\title{
ORDER AND NORM CONVERGENCE IN BANACH LATTICES
}

\author{
by ANDREW WIRTH†
}

(Received 27 June, 1972; revised 19 September, 1972)

Let $(V, \leqq,\|\cdot\|)$ be a Banach lattice, and denote $V\{0\}$ by $V^{\prime}$. For the definition of a Banach lattice and other undefined terms used below, see Vulikh [4]. Leader [3] shows that, if norm convergence is equivalent to order convergence for sequences in $V$, then the norm is equivalent to an $M$-norm. By assuming the equivalence for nets in $V$ we can strengthen this result.

THEOREM. Let $(V, \leqq,\|\cdot\|)$ be a Banach lattice; then the following statements are equivalent:

(i) Norm convergence is equivalent to order convergence, for nets in $V$.

(ii) Vis finite-dimensional.

Proof. (i) implies (ii). If $\alpha, \beta \in V^{\prime}$, write $\alpha \preccurlyeq \beta$ to mean $\|\alpha\| \geqq\|\beta\|$. Then $\left(V^{\prime}, \preccurlyeq\right)$ is a preordered set directed to the right. Let $x_{\alpha}=\alpha$ for all $\alpha \in V^{\prime}$; then $\|\cdot\|-\lim x_{\alpha}=0$, and so 0 -lim $x_{\alpha}=0$. Hence $(V, \leqq)$ has a strong unit, $e$ say. Define $\|\cdot\|_{e}$ by $\|x\|_{e}=\inf \{\lambda:|x| \leqq \lambda e\}$, for $x \in V$. By Birkhoff [1], $\|\cdot\|$ and $\|\cdot\|_{e}$ are equivalent norms. In fact $\left(V, \leqq,\|\cdot\|_{e}\right)$ is a Banach lattice with unity $e$ and so an $M$-space, Birkhoff [1]. So $\left(V, \leqq,\|\cdot\|_{e}\right)$ is isomorphic with $(C(X), \leqq$, sup norm), $X$ compact Hausdorff, by Kelley and Namioka [2].

Let $x_{0} \in X$ and let $g$ be the characteristic function for the point $x_{0}$. Define

$$
F=\left\{f \in C(X): f \geqq 0 \text { and } f\left(x_{0}\right)=1\right\} ;
$$

then $(F, \geqq)$ is directed to the right. Let $f_{\alpha}=\alpha$ for all $\alpha \in F$. Then, by Urysohn's Lemma, $f_{\alpha} \downarrow g$ pointwise. If $g \in C(X)$, then 0 - $\lim f_{\alpha}=g$; otherwise 0 - $\lim f_{\alpha}=0$. Now $\|\cdot\|_{e}-\lim f_{\alpha}=0$ is impossible; so $g \in C(X)$. Hence $\left\{x_{0}\right\}$ is open; so $X$ is discrete and hence finite.

(ii) implies (i). The proof of this is trivial.

The author is grateful to the referee for suggesting an improvement to the paper.

\section{REFERENCES}

1. G. Birkhoff, Lattice Theory, Amer. Math. Soc. Colloquium Publications 25, 3rd Edition (Providence, R. I., 1967), 366-377.

2. J. L. Kelley and I. Namioka, Linear topological spaces (Princeton, 1963), 236-242.

3. S. Leader, Sequential convergence in lattice groups, Problems in Analysis (ed. R. C. Gunning), (Princeton, 1970), 273-290.

4. B. Z. Vulikh, Introduction to the theory of partially ordered spaces (Groningen, 1967).

MONASH UNIVERSITY

Clayton, Victoria, Australia, 3168

$\dagger$ This research was supported by a Commonwealth Postgraduate Research Award. 